\title{
Paraoxonase 1 Activity and Survival in Sepsis Patients
}

\author{
Volkan İnal' ${ }^{1}$, Levent Yamanel${ }^{2}$, Gürhan Taşkın², Serkan Tapan ${ }^{3}$, Bilgin Cömert ${ }^{4}$
}

${ }^{1}$ Department of Critical Care, Trakya University Faculty of Medicine, Edirne, Turkey ${ }^{2}$ Department of Critical Care, Gülhane Military Academy of Medicine, Ankara, Turkey ${ }^{3}$ Department of Biochemistry, Gülhane Military Academy of Medicine, Ankara, Turkey

${ }^{4}$ Department of Critical Care, Dokuz Eylül University Faculty of Medicine, İzmir, Turkey

Background: Sepsis is a state of augmented oxidative stress and diminished antioxidant capacity. High density lipoprotein (HDL) particles were shown to possess antioxidant and anti-inflammatory properties, as well as Paraoxonase 1 (PON1), which is an enzyme that is also protective against HDL oxidation. Previous studies suggested a possible role of decreased PON1 activity or HDL levels in sepsis patients.

Aims: The present study was designed to test a hypothesis that higher PON1 activity and HDL-cholesterol levels could predict a better survival in sepsis patients. Study Design: Observational study.

Methods: Venous blood samples were collected from sepsis patients for HDL-cholesterol levels, PON1 activity and cytokine assays (TNF- $\alpha$ and IL- 6 ) and Acute Physiologic and Chronic Health Evaluation II (APACHE II) scores were calculated in order to weight patients' disease severity on the day of sepsis diagnosis. Patients were followed-up until the $28^{\text {th }}$ day for any cause intrahospital mortality. Data were statistically analyzed for effects of study parameters on patients' survival.

Results: In total, 85 patients with sepsis were included in the study. The mean age was $65.2 \pm 17.9$ years and 48 were male; at the end of the 28-day follow-up pe- riod, 46 survived. TNF- $\alpha(86.9 \pm 10.5$ vs $118.6 \pm 16.4)$ and IL-6 levels $(906.7 \pm 82.7$ vs $1323.1 \pm 54.3)$ were significantly higher in non-survivors, while PON1 activity (140.7 \pm 42.3 vs $66.7 \pm 46.6)$ and HDL-cholesterol levels (43.6 \pm 8.1 vs $34.5 \pm 8.9)$ were significantly higher in survivors $(\mathrm{p}<0.001$ for all $)$ TNF- $\alpha(\mathrm{r}=-0.763)$ and IL-6 levels $(\mathrm{r}=-0.947)$ showed strong negative correlations, PON1 activity $(\mathrm{r}=0.644)$ and HDL-cholesterol levels $(r=0.477)$ showed positive correlations with patient survival ( $p<0.001$ for all). Survival estimates significantly favored TNF- $\alpha$ (Log Rank 59.5, $\mathrm{p}<0.001)$ and IL-6 levels (Log Rank 53.2, $\mathrm{p}<0.001$ ) according to PON1 activity (Log Rank 5.4, $\mathrm{p}<0.03$ ) and HDL-cholesterol levels (Log Rank 8.3, $\mathrm{p}<0.005$ ). Regression analyses for relative contributions of parameters to survival showed that higher IL-6 levels $(\mathrm{t}:-16.489, \mathrm{p}<0.001)$ were the most significant negative factor for survival, and TNF- $\alpha$ levels ( $\mathrm{t}:-4.417, \mathrm{p}<0.001)$, whereas PON1 activity had a positive effect $(\mathrm{t}: 3.210, \mathrm{p}<0.003)$.

Conclusion: The present study showed that although low PON1 activity and HDL-cholesterol levels were related to mortality, higher levels were not found to be as predictive as cytokine levels for survival.

Keywords: Mortality, paraoxonase 1, sepsis
Sepsis is a systemic inflammatory response triggered by an infection (1). In the first phase of sepsis, inflammatory mediator surge by defense mechanisms causes an increased oxidative stress state (2). The second phase is defined by an anti-inflammatory surge while the inflammatory process con- tinues. The final outcome is determined by the challenge of inflammatory and anti-inflammatory mechanisms to regain a balanced-state (3). Dysregulation of this desired balance toward the inflammatory and oxidative state is accused for the undesired destructive outcomes (4). 
Previous studies have considered anti-inflammatory and anti-oxidant effects of Paraoxonase 1 (PON1) and high density lipoproteins (HDL). Decreased PON1 activity and HDL levels were shown in sepsis. In addition, the inflammatory condition caused oxidation of HDL particles, which in turn acted as a pro-inflammatory mediator (5-8). PON1 is an enzyme that is synthesized primarily in the liver, which metabolizes toxic oxidized HDL particles, and plays an anti-inflammatory role in inflammation (9). HDL particles and PON1 have been considered to neutralize lipopolysaccharides (LPS), inhibit the synthesis of some pro-inflammatory cytokines and modulate endothelial function $(5,10)$.

Assuming the possible relations in the sepsis process, the present study was designed to test a hypothesis that higher PON1 activity and HDL-cholesterol levels could predict a better survival in sepsis patients.

\section{MATERIALS AND METHODS}

The study was designed as an observational study, and performed on patients in a medical intensive care unit (ICU) of a university research hospital, within a two month period. The ethics committee approval was received from the local authority. Participants were included in the study after their written informed consent was received.

\section{Patients and study design}

The patients in the ICU were screened for sepsis, based on the definition of the American College of Chest Physicians/ Society of Critical Care Medicine Consensus Conference, and were treated as recommended (1). Patients with end-stage cancer, liver cirrhosis, history of hyperlipidemia, on lipidlowering drugs or receipt of total parenteral nutrition were not included in the study, to avoid bias on PON1 and HDL-cholesterol levels. Venous blood samples were collected on the day of sepsis diagnosis from all participating patients for measurements of HDL-cholesterol levels and PON1 activities and cytokine assays (TNF- $\alpha$ and IL-6 levels). The first day Acute Physiologic and Chronic Health Evaluation II (APACHE II) scores, which contains 14 parameters of patient's status and have been shown to be predictive for patient survival in the ICU, were calculated in order to weight patients' disease severity and mortality estimation (11). Patients were followedup for survival or any-cause intra-hospital mortality until the $28^{\text {th }}$ day.

\section{Biochemical measurements}

An auto-analyzer (Hitachi 917 Clinical Immunoassay Analyzer; Roche Diagnostics, Indianapolis, U.S.) was used for HDL-cholesterol assays (mg/dL).

\section{Paraoxonase 1 activity assay}

Paraoxonase 1 activity in plasma was measured using paraoxon (Paraoxon; Sigma-Aldrich Co. LLC., Taufkirchen, Germany) as a substrate, and the assay was conducted at a temperature of $37^{\circ} \mathrm{C}$ in a microplate reader (BioTech ${ }^{\circledR}$ Synergy ${ }^{\mathrm{TM}} \mathrm{HT}$ Reader; BioTech $^{\circledR}$ Synergy ${ }^{\mathrm{TM}}$, Vermont, U.S.). An aliquot of plasma (10 $\mu \mathrm{L})$ was resuspended in a basal assay mixture $(5 \mathrm{mM}$ Tris- $\mathrm{HCl}$, pH 7.4 containing $0.15 \mathrm{M} \mathrm{NaCl}, 4 \mathrm{mM} \mathrm{MgCl}, 2 \mathrm{mM} \mathrm{CaCl}$ and $1.0 \mathrm{mmol} / \mathrm{L}$ paraoxon). Paraoxon hydrolysis was kinetically monitored for 8 minutes (every 15 seconds) at $412 \mathrm{~nm}$. The non-enzymatic hydrolysis of paraoxon was subtracted from the total rate of hydrolysis. The enzyme activity was calculated by $\mathrm{KC}^{\mathrm{TM}}$ software $\left(\mathrm{KC}^{\mathrm{TM}}{ }^{\mathrm{TM}}\right.$; BioTech ${ }^{\circledR}$ Synergy $^{\mathrm{TM}}$, Vermont, U.S.) for microplate reader $(\mathrm{U} / \mathrm{mL})$ as described by Huen et al (12).

\section{Cytokine assays}

Samples were allowed to clot for 30 minutes before centrifugation for 15 minutes at approximately $1000 \mathrm{x} \mathrm{g}$. After centrifugation, serum was immediately stored at $-80^{\circ} \mathrm{C}$. TNF- $\alpha$ and IL- 6 in serum samples were measured by quantitative sandwich ELISA kits (Human TNF- $\alpha$ Quantikine ELISA Kit and Human IL-6 Quantikine ELISA Kit; R\&D System Inc., Minneapolis, U.S.) (pg/mL).

\section{Statistical analysis}

The required sample size was estimated by a software package (G-Power ${ }^{\odot}$ 3.0.10 PC; by Fraz Faul, Universtat Kiel, Kiel, Germany) and calculated to 82 (effect size 0.5 , two-tailed, $\alpha$ 0.05 , power 0.8 ). Statistical analyses were performed using SPSS (SPSS PC Ver.22; IBM ${ }^{\circ}$ SPSS Inc., New York, U.S.). Data were presented as mean $\pm \mathrm{SD}$ and two-tailed probabilities less than 0.05 with $95 \%$ CI were considered as significant. Parametric data were tested for distribution normality; Student's T test and Pearson correlation $(r)$ were performed when appropriate. The ability of study parameters to predict the 28day mortality rate was analyzed by generating Receiver Operator Characteristics (ROC) and Area under Curve (AUC). Kaplan-Meier survival analysis (28 day), Linear Regression analyses and Automatic Linear Modelling (ALM) were performed to show the possible effects of parameters on survival, all weighted by APACHE II scores. Automatic Linear Modelling was a specific tool for modelling larger studies, using the SPSS software, which helped to estimate the relative contributions of factors on the target.

\section{RESULTS}

According to the predefined criteria of the study, 85 patients with sepsis were included in the study. The mean age 
TABLE 1. The descriptive (mean \pm SD) values of study parameters and statistical comparison by Student's T test

\begin{tabular}{llcc}
\hline & & mean \pm SD & Student's T \\
\hline APACHE II score & $\mathrm{s}$ & $20.9 \pm 2.9$ & $\mathrm{p}<0.001$ \\
& $\mathrm{~ns}$ & $28.1 \pm 2.8$ & \\
TNF- $\alpha(\mathrm{pg} / \mathrm{mL})$ level & $\mathrm{s}$ & $86.9 \pm 10.5$ & $\mathrm{p}<0.001$ \\
& $\mathrm{~ns}$ & $118.6 \pm 16.4$ & \\
IL-6 (pg/mL) level & $\mathrm{s}$ & $906.7 \pm 82.7$ & $\mathrm{p}<0.001$ \\
& $\mathrm{~ns}$ & $1323.1 \pm 54.3$ & \\
PON1 (U/mL) activity & $\mathrm{s}$ & $140.7 \pm 42.3$ & $\mathrm{p}<0.001$ \\
& $\mathrm{~ns}$ & $66.7 \pm 46.6$ & \\
HDL-C (mg/dL) level & $\mathrm{s}$ & $43.6 \pm 8.1$ & $\mathrm{p}<0.001$ \\
& $\mathrm{~ns}$ & $34.5 \pm 8.9$ & \\
\end{tabular}

APACHE II: Acute Physiologic and Chronic Health Evaluation II; TNF- $\alpha$ : Tumor Necrosis Factor-alpha; IL-6: Interleukin-6; PON1: Paraoxonase 1; HDL-C: HDLcholesterol; (s): Survivors; (ns): Non-Survivors

TABLE 2. Pearson correlation and ROC analyses data for survival

\begin{tabular}{lcc}
\hline & Pearson Correlation & ROC \\
\hline IL-6 $(\mathrm{pg} / \mathrm{mL})$ level & $\mathrm{r}=(-) 0.947$ & AUC: $1.0 \mathrm{CI}: 1.0-1.0$ \\
& $\mathrm{p}<0.001$ & $\mathrm{p}<0.001$ \\
TNF- $\alpha(\mathrm{pg} / \mathrm{mL})$ level & $\mathrm{r}=(-) 0.763$ & AUC:0.941 CI:0.895-0.986 \\
& $\mathrm{p}<0.001$ & $\mathrm{p}<0.001$ \\
PON1 (U/mL) activity & $\mathrm{r}=0.644$ & AUC:0.874 CI:0.788-0.960 \\
& $\mathrm{p}<0.001$ & $\mathrm{p}<0.001$ \\
HDL-C (mg/dL) level & $\mathrm{r}=0.477$ & AUC:0.776 CI:0.671-0.881 \\
& $\mathrm{p}<0.001$ & $\mathrm{p}<0.001$ \\
\hline
\end{tabular}

ROC: Receiver Operator Characteristics; AUC: Area under Curve; IL-6: Interleukin-6; TNF- $\alpha$ : Tumor Necrosis Factor-alpha; PON1: Paraoxonase 1; HDL-C: HDL-cholesterol

was $65.2 \pm 17.9$ years and 48 were male; at the end of 28-day follow up period, 46 had survived. There were no statistical difference between surviving and deceased patients in respect to age and gender.

APACHE II scores, and TNF- $\alpha$ and IL-6 levels were significantly higher in non-survivors; on the other hand, PON1 activity and HDL-cholesterol levels were significantly higher in survivors ( $\mathrm{p}<0.001$ for all parameters) (Table 1).

Correlation and ROC analyses data for patients' survival or death are presented in Table 2. TNF- $\alpha$ and IL-6 levels showed strong negative correlations with patient survival ( $p<0.001$ for both). Although both were statistically significant, PON1 activity and HDL-cholesterol levels showed positive but weaker correlations with patient survival than cytokines $(\mathrm{p}<0.001$ for both). Similar results were found in ROC-AUC analyses for predictive power of these parameters for patient's survival or death.

The results of Kaplan-Meier survival analysis also significantly favored TNF- $\alpha$ and IL-6 levels in short-mid-long
TABLE 3. Kaplan-Meier survival analyses by factor APACHE II scores

\begin{tabular}{lccc}
\hline & Log Rank $\chi^{2}$ & Breslow $\chi^{2}$ & Tarone-Ware $\chi^{2}$ \\
\hline TNF- $\alpha(\mathrm{pg} / \mathrm{mL})$ level & 59.498 & 48.106 & 53.684 \\
& $(\mathrm{p}=0.000)$ & $(\mathrm{p}=0.000)$ & $(\mathrm{p}=0.000)$ \\
IL-6 $(\mathrm{pg} / \mathrm{mL})$ level & 53.240 & 44.762 & 49.111 \\
& $(\mathrm{p}=0.000)$ & $(\mathrm{p}=0.000)$ & $(\mathrm{p}=0.000)$ \\
HDL-C $(\mathrm{mg} / \mathrm{dL})$ level & 8.349 & 7.408 & 8.014 \\
& $(\mathrm{p}=0.004)$ & $(\mathrm{p}=0.006)$ & $(\mathrm{p}=0.005)$ \\
PON1 $(\mathrm{U} / \mathrm{mL})$ activity & 5.382 & 3.132 & 4.135 \\
& $(\mathrm{p}=0.020)$ & $(\mathrm{p}=0.077)$ & $(\mathrm{p}=0.042)$ \\
\hline APACHE II: Acute Physiologic and Chronic Health Evaluation II; TNF- $\alpha$ : Tumor \\
Necrosis Factor-alpha; IL-6: Interleukin-6; HDL-C: HDL-cholesterol; PON1: \\
Paraoxonase 1
\end{tabular}

TABLE 4. Regression analyses summary; estimates of relative contributions of factors for survival weighted by APACHE II scores, and Automatic Linear Modelling (ALM) estimates for relative importance (CI: 95\%, accuracy: 93.4\%)

\begin{tabular}{lcc}
\hline & $(\mathrm{t}) \mathrm{p}$ & (ALM) importance \\
\hline IL-6 $(\mathrm{pg} / \mathrm{mL})$ level & $(\mathrm{t}:-16.489) \mathrm{p}=0.000$ & 0.889 \\
TNF- $\alpha(\mathrm{pg} / \mathrm{mL})$ level & $(\mathrm{t}:-4.417) \mathrm{p}=0.000$ & 0.070 \\
PON1 $(\mathrm{U} / \mathrm{mL})$ activity & $(\mathrm{t}: 3.210) \mathrm{p}=0.002$ & 0.004 \\
\hline
\end{tabular}

APACHE II: Acute Physiologic and Chronic Health Evaluation II; IL-6: Interleu-

kin-6; TNF- $\alpha$ : Tumor Necrosis Factor-alpha; PON1: Paraoxonase 1

term estimates ( $\mathrm{p}<0.001$ for both). In addition, PON1 activity and HDL-cholesterol levels were also statistically significant ( $\mathrm{p}<0.001$ for both) (Table 3).

Regression analyses for relative contributions of study parameters to survival weighted by APACHE II scores showed that higher IL-6 levels were the most significant negative factor for survival $(p<0.001)$. TNF- $\alpha$ levels were also a significant negative factor $(\mathrm{p}<0.001)$, whereas PON1 activity had a positive but less significant effect $(\mathrm{p}<0.003)$. Automatic Linear Modelling (ALM) estimates, also weighted by APACHE II scores, for relative importance of parameters for survival indicated that IL-6 levels had the highest importance, while PON1 activity had the least importance among parameters (CI: 95\%, test accuracy: 93.4\%) (Table 4).

\section{DISCUSSION}

Sepsis is one of the leading causes of mortality in ICUs, despite recent diagnostic and therapeutic advances (1). Novel accumulating evidences on pathophysiologic processes in sepsis have encouraged researchers to conduct studies on different aspects of the inflammatory cascade. The present study aimed to test possible effects of known antioxidant and antiinflammatory properties of HDL-cholesterol and PON1 activity on sepsis survival. Considerable changes in the concentration and composition of plasma lipids and lipoproteins have 
been reported in patients with sepsis (13). Concentrations of lipoproteins, especially high density lipoproteins, were markedly reduced and these changes were found to be closely related to the severity of infection (10).

Shor et al. (14) compared 108 patients with HDL-cholesterol levels of $\leq 20 \mathrm{mg} / \mathrm{dL}$ and 96 patients with $\geq 65 \mathrm{mg} / \mathrm{dL}$, and reported that lower HDL-cholesterol levels were associated with higher mortality rates (OR 0.96, 95\% 0.93-0.99, $\mathrm{p}=0.02$ ). They observed that for each $1 \mathrm{mg} / \mathrm{dL}$ increase in HDL-cholesterol levels, a relative $11 \%$ decrease in odds of sepsis mortality occurred. In our study, HDL-cholesterol levels were also significantly higher in survivors. On the other hand, our nonsurvivors' HDL-cholesterol levels were higher $(34.5 \pm 8.9 \mathrm{mg} /$ $\mathrm{dL})$ than the non-survivor HDL-cholesterol levels $(16.1 \pm 33$ $\mathrm{mg} / \mathrm{dL}$ ) in the study by Shor. That was possibly due to differences between study populations and designs, as they included patients with malignancies, all patients were not suffering sepsis and they used a different disease weighting scale than APACHE-II, which was used in our study. In addition, they specifically tested effect mortality but not survival, and their survivor group HDL-cholesterol levels were much higher $(74.9 \pm 12.6 \mathrm{mg} / \mathrm{dL})$ than those of our survivors $(43.6 \pm 8.1 \mathrm{mg} /$ $\mathrm{dL}$ ); also, our whole patient population was in sepsis. In other words, comparison of their results with ours was not totally adaptable.

Barlage et al. (15) compared lower levels of HDL-cholesterol with 30-day-mortality on 151 sepsis patients. They used a 12-variable disease severity scale SAPS II (Simplified Acute Physiology Score). On the other hand, they did not prefer weighting study variables for SPAS II in order to stratify predicted disease mortality risk, as we did by APACHE II; consequently, regression analyses inevitably found SAPS II to be the best predictor. We assumed that these already-approved disease scoring systems should be used to weight patients' status, as they would down-scale the power of other variables, as stated by Barlage et al. (15). Besides, they showed significantly $(p=0.007)$ higher HDL-cholesterol levels in survivors, as in our study, but did not mention a predictive power of the finding.

In recent years, a controlled study on 29 sepsis and 41 nonsepsis patients in ICU mentioned that HDL-cholesterol levels were significantly lower in the sepsis group $(20 \pm 5.6 \mathrm{mg} / \mathrm{dL}$ vs $30.2 \mathrm{mg} / \mathrm{dL}, \mathrm{p}<0.01$ ), but did not correlate with mortality rates, and no regression model was applied to propose any prognostic effect (13). They also did not weighted patients' disease severity by any score. As a limitation of our study, we did not have a control group; instead, we compared levels in survivors and non-survivors. In our study, HDL-cholesterol levels in non-survivors were also found to be lower than survivors, and higher levels were predictive for survival in regression.
Besides, HDL studies on sepsis showed lower levels associated with higher mortality rates and no evidence of higher levels on survival; as in our study, experimental studies on PON1 activity also showed decreased levels in sepsis models $(16,17)$. Increased serum oxidative stress and HDL oxidation has been demonstrated in PON1-deficient mice (18). These preliminary results assigned a possible protective role of PON1 activity and consequently encouraged novel studies.

Novak et al. (19), in a controlled study, found lower PON1 activities in sepsis patients than in healthy controls $(75.2 \pm 50.0$ vs. $125.2 \pm 69.4 \mathrm{U} / 1, \mathrm{p}<0.01, \mathrm{n}=30 / 30)$ and PON1 activities normalized one week after patients' recovery. A positive correlation was also shown between HDL-cholesterol and PON1 activity ( $r=0.405, p<0.01)$. Novak's study was similar to ours with respect to that, as it used the APACHE II score for sepsis patients, a standard sepsis definition, and patient exclusion criteria. Otherwise, it was controlled study; they resampled PON1 activities one week after patient recovery, and the mortality rates (6 of 30) presented were lower than ours (39 of 85). In contrast, the lack in resampling of PON1 activities was another limitation of our study, as we did not analyze later values after patient recovery. Besides, the higher mortality rates in our study could be explained by their mean APACHE II scores being lower than those of non-survivors in our study (20.1 \pm 6.8 vs. $28.1 \pm 2.8)$, on the other hand APACHE II scores of survivors were similar in our and Nocak's study (20.1 \pm 6.8 vs. 20.9 \pm 2.9 ). On the contrary to our results, they found no correlation between survivors' and non-survivors' PON1 activities, possibly due to their sepsis population being smaller than ours and the fact that they did not weight by APACHE II scores. In addition, they did not present a regression analysis to predict for survivors. However, we carried out regression analyses and weighted by APACHE II, but we were not able to demonstrate a strong correlation between PON1 activities and patients' survival, although the results were statistically significant, but with weaker power.

In a controlled study, Li et al. (8) compared PON1 activity between sepsis patients $(n=61)$ and healthy controls $(n=32)$ to predict 30 -day mortality rates. Mortality rate was $39 / 61$, and PON1 activity was found significantly lower in non-survivors $(104.9 \pm 23.0$ vs $85.1 \pm 16.6 \mathrm{U} / 1, \mathrm{p}=0.001)$, which was similar to our results. This study was also similar in respect to patient inclusion criteria, follow-up period, preferred disease scoring scale (APACHE II), and survival analysis, and was also a controlled study which compared healthy controls. Sepsis patients' APACHE II scores (17.5 \pm 2.1 vs $20.1 \pm 2.3$, survivors vs non-survivors) were lower than our patients' scores, possibly confounding mortality risk prediction, and no cytokine assay was done that could change the effect of parameters on outcome in regression analysis. In our study, ROC and the 
correlation analysis of PON1 activity for survivors were also statistically significant ( $<0.001$ for both), but the relatively low power of these values ( $\mathrm{r}=0.644$, AUC: 0.874$)$ in respect to IL- 6 and TNF- $\alpha$ values should be noted. In our study, PON1 activity $(\mathrm{p}=0.020)$ power was lower than that of $\mathrm{Li}(\mathrm{p}=0.0013)$ when survival was analyzed by factor "APACHE II score". On the contrary, when survival was analyzed without the factor by "APACHE II score" it gained higher significance $(\mathrm{p}<0.001)$. We criticized this difference as a possible bias because Li ignored patients' "APACHE II scores", and also the patients' disease severity.

In a recent controlled study, Bojic et al. (7) compared PON1 activity in sepsis patients with matched healthy controls $(n=69 / 69)$ for survival. They measured PON1 activities upon admission to ICU, and then in the $24^{\text {th }}, 48^{\text {th }}$, $72^{\text {th }}$ and $96^{\text {th }}$ hours. Septic patients had significantly lower PON1 activities compared to the control group at alltime points, and PON1 activities showed a good capacity to differentiate septic patients from healthy controls. Low PON1 activities were associated with higher disease severity scores and a higher risk of death, as in our study. ROC analysis results used to evaluate PON1 activity as a marker were similar to ours (AUC: $0.856,95 \%$ CI: $0.777-0.934$ vs AUC: $0.874, \% 95$ CI: $0.788-0.960, p<0.001$ for both); on the other hand, they did not show a correlation between PON1 activities and inflammatory markers. They used, but did not weight by, APACHE II scores as a parameter, and found significant results for PON1 activity with KaplanMeier survival analysis (Log Rank $\mathrm{p}<0.001)$. On the other hand, in our study, survival estimates were not as significant for PON1 activities as found by other groups ( $\mathrm{Log}$ Rank $\mathrm{p}<0.03)$. We criticized this difference as we weighted patients' parameters by APACHE II scores; otherwise, it produced an overestimated high significance $(\mathrm{p}<0.001)$, and did not reflect the patient-specific situation. Indeed, they did not conclude PON1 to be a prognostic marker, but confirmed that it showed disease severity.

In conclusion, the present study has shown that lower PON1 activity and HDL-cholesterol levels were related to higher mortality rates in sepsis patients, but higher levels could not definitely predict patient survival better than cytokine levels. Thus, future studies on PON1 activity and HDL-cholesterol levels in sepsis patients are still required.

Ethics Committee Approval: Ethics committee approval was received for this study from the ethics committee of the local ethic committee of Gülhane Military Academy of Medicine (protocol number: 50687469-1491-352-14/1648.4-1054; 13.05.2014).

Informed Consent: Written informed consent was obtained from patients who participated in this study.
Peer-review: Externally peer-reviewed.

Author contributions: Concept - V.İ., B.C.; Design - B.C.; Supervision - L.Y., B.C.; Resource -S.T.; Materials - V.İ., G.T.; Data Collection \&/or Processing - V.İ., G.T., S.T.; Analysis \&/or Interpretation - V.İ., S.T., B.C.; Literature Search - V.İ., G.T., B.C.; Writing - V.İ., L.Y.; Critical Reviews - V.İ., L.Y., G.T., S.T., B.C.

Conflict of Interest: No conflict of interest was declared by the authors.

Financial Disclosure: The authors declared that this study has received no financial support.

\section{REFERENCES}

1. Dellinger RP, Levy MM, Rhodes A, Annane D, Gerlach H, Opal $\mathrm{SM}$, et al. Surviving sepsis campaign: international guidelines for management of severe sepsis and septic shock: 2012. Crit Care Med 2013;41:580-637. [CrossRef]

2. Galley HF. Oxidative stress and mitochondrial dysfunction in sepsis. Br J Anaesth 2011;107:57-64. [CrossRef]

3. Pinsky MR. Dysregulation of the immune response in severe sepsis. Am J Med Sci 2004;328:220-9. [CrossRef]

4. Andrades ME, Ritter C, Dal-Pizzol F. The role of free radicals in sepsis development. Front Biosci (Elite Ed) 2009;1:277-87.

5. Draganov D, Teiber J, Watson C, Bisgaier C, Nemzek J, Remick $\mathrm{D}$, et al. PON1 and oxidative stress in human sepsis and an animal model of sepsis. Adv Exp Med Biol 2010;660:89-97. [CrossRef]

6. Chien JY, Jerng JS, Yu CJ, Yang PC. Low serum level of highdensity lipoprotein cholesterol is a poor prognostic factor for severe sepsis. Crit Care Med 2005;33:1688-93. [CrossRef]

7. Bojic S, Kotur-Stevuljevic J, Kalezic N, Jelic-Ivanovic Z, Stefanovic A, Palibrk I, et al. Low paraoxonase 1 activity predicts mortality in surgical patients with sepsis. Dis Markers 2014;2014:427378. [CrossRef]

8. Li Y, Zhai R, Li H, Mei X, Qiu G. Prognostic value of serum paraoxonase and arylesterase activity in patients with sepsis. $J$ Int Med Res 2013;41:681-7. [CrossRef]

9. Mackness B, Mackness M. Anti-inflammatory properties of paraoxonase-1 in atherosclerosis. Adv Exp Med Biol 2010;660:14351. [CrossRef]

10. Barter PJ, Nicholls S, Rye KA, Anantharamaiah GM, Navab M, Fogelman AM. Antiinflammatory properties of HDL. Circ Res 2004;95:764-72. [CrossRef]

11. Knaus WA, Draper EA, Wagner DP, Zimmerman JE. APACHE II: a severity of disease classification system. Crit Care Med 1985;13:818-29. [CrossRef]

12. Huen K, Richter R, Furlong C, Eskenazi B, Holland N. Validation of PON1 enzyme activity assays for longitudinal studies. Clinic Chimic Acta 2009;402:67-74. [CrossRef]

13. Barati M, Nazari MR, TalebiTaher M, Farhadi N. Comparison of Lipid Profile in Septic and Non-Septic Patients. Iran J Clin Infect Dis 2011;6:144-47. 
14. Shor R, Wainstein J, Oz D, Boaz M, Matas Z, Fux A, et al. Low HDL levels and the risk of death, sepsis and malignancy. Clin Res Cardiol 2007;97:227-33. [CrossRef]

15. Barlage S, Gnewuch C, Liebisch G, Wolf Z, Audebert FX, Glück T, et al. Changes in HDL-associated apolipoproteins relate to mortality in human sepsis and correlate to monocyte and platelet activation. Intensive Care Med 2009;35:1877-85. [CrossRef]

16. Costa LG, Furlong CE. Paraoxonase (PON1) in health and disease: basic and clinical aspects. Ch.6. Protective action of HDLassociated PON1 against LDL oxidation, Ch.Ed; Navab M.,
Hama S.Y., Wagner A.C., Hough G., Watson A.D.), pp. 125-36. Norwell, MA: Kluwer Academic Publishers; 2002.

17. Feingold KR, Memon RA, Moser AH, Grunfeld C. Paraoxonase activity in the serum and hepatic mRNA levels decrease during the acute phase response. Atherosclerosis 1998;139:307-15. [CrossRef]

18. Shih DM, Gu L, Xia YR, Navab M, Li WF, Hama S, et al. Mice lacking serum paraoxonase are susceptible to organophosphate toxicity and atherosclerosis. Nature 1998;394:284-7. [CrossRef]

19. Novak F, Vavrova L, Kodydkova J, Sr FN, Hynkova M, Zak A, et al. Decreased paraoxonase activity in critically ill patients with sepsis. Clin Expe Med 2010;10:21-5. [CrossRef] 